\section{Depressão na esquizofrenia: prevalência e relação com a qualidade de vida}

\author{
Depression in schizophrenia: prevalence and \\ relationship to quality of life
}

\author{
1 Faculdade de Medicina, \\ Universidade Federal de \\ Minas Gerais, Belo Horizonte, \\ Brasil. \\ 2 Grupo de Pesquisa em \\ Epidemiologia, Universidade \\ Federal de Minas Gerais, \\ Belo Horizonte, Brasil. \\ 3 Departamento de \\ Psicologia, Universidade \\ Federal de São João Del-Rei, \\ São João Del-Rei, Brasil. \\ ${ }^{4}$ Departamento de \\ Estatística, Universidade \\ Federal de Minas Gerais, \\ Belo Horizonte, Brasil. \\ Correspondência \\ C. S. Cardoso \\ Grupo de Pesquisa em \\ Epidemiologia Universidade \\ Federal de Minas Gerais. \\ Av. Alfredo Balena 190, \\ sala 8013, Belo Horizonte MG \\ 30130-100, Brasil. \\ clarecicardoso@yahoo.com.br
}

\section{Abstract}

This study aimed to investigate the prevalence of depression in schizophrenia and associated factors, including quality of life. A cross-sectional study was conducted with 150 outpatients. The Calgary Depression Scale for Schizophrenia measured depression, and the Quality of Life Scale-Brazil measured quality of life. Major depression was found in 56\% of patients with schizophrenia. Patients with major depression had low quality of life according to both the global scale and the occupational subscale. Global quality of life was important for separating the decision-tree statistical analyses. In patients with low quality of life, three factors were associated with depression: presence of schizophrenic symptoms, number of medications, and lack of household activities. In patients with better quality of life scores, only duration of the illness was significant. The study shows a high prevalence of depression in schizophrenia, besides highlighting its repercussions on quality of life. When evaluating quality of life, the presence of depression should be taken into consideration.

Depression; Schizophrenia; Quality of Life

\author{
Clareci Silva Cardoso 1,2 \\ Waleska Teixeira Caiaffa 1,2 \\ Marina Bandeira 3 \\ Arminda Lucia Siqueira 4 \\ Jussara Teixeira da Silva 2 \\ José Otávio Penido Fonseca 1,2
}

\section{Introdução}

Sabe-se que a presença de sintomas depressivos tem estreita associação com a baixa qualidade de vida em pacientes com esquizofrenia 1 . Nesses pacientes, estudos têm dado atenção especial a essa morbidade, que se apresenta como indicador de prognóstico desfavorável ${ }^{2}$, associado a vários aspectos negativos do desfecho clínico, tais como aumento do risco de suicídio 3,4 , piora no funcionamento psicossocial 5 , aumento de recaídas, hospitalizações mais prolongadas, pior resposta à medicação e cronicidade 6 .

Estudos de qualidade de vida relatam uma importante associação entre depressão e qualidade de vida na esquizofrenia 1,7,8,9. Pacientes deprimidos apresentam-se menos satisfeitos com suas atividades diárias, com a saúde física e mental, além de pior qualidade de vida global, independente da presença de sintomatologia da doença 1,10 .

Em estudos internacionais, a prevalência de depressão na esquizofrenia varia de $7 \%$ a $70 \% 11$, com média de $25 \%$ 12. Para o Brasil foram encontradas taxas em torno de $27 \%$ e $29,8 \%$ 13,14,15 Outros dados da literatura relatam que, cerca de $60 \%$ dos pacientes com esquizofrenia desenvolveriam pelo menos um episódio depressivo maior, ao longo de sua doença 16. Embora de grande relevância clínica, estudos indicam que a metade dos casos de depressão em nível primário de saúde não são detectados 9 . 
A avaliação dos sintomas depressivos na esquizofrenia esteve, por muito tempo, restrito exclusivamente ao período pós-psicótico. Entretanto, estudos mais recentes demonstram que eles podem ocorrer em qualquer fase da doença 17,18,19. Seu diagnóstico foi, por muito tempo, dificultado pela sobreposição com os sintomas negativos ou extrapiramidais induzidos pelos neurolépticos, constituindo um problema freqüente. Entretanto, devido à alta prevalência da depressão e seu impacto psicossocial, foram surgindo instrumentos especificamente planejados para mensurar sua gravidade e auxiliar na identificação desta condição, reduzindo consideravelmente as dificuldades diagnósticas. Dentro dessa perspectiva, a Escala Calgary de Depressão na Esquizofrenia (ECDE) tem sido amplamente utilizada, possibilitando a avaliação da depressão independentemente da sintomatologia negativa ou dos sintomas induzidos pelos neurolépticos. Essa escala foi validada para o Brasil e apresentou características adequadas de validade e confiabilidade 14,20.

Embora a literatura tenha mostrado evidências do impacto da sintomatologia depressiva na qualidade de vida dos pacientes com esquizofrenia, não foram identificados trabalhos brasileiros investigando a qualidade de vida sob a ótica da depressão. Este conhecimento é considerado pertinente, tendo em vista que as intervenções terapêuticas e medicamentosas podem melhorar a qualidade de vida desta população, quando a sintomatologia depressiva é previamente identificada 21 . Portanto, além de prevalente e subdiagnosticada, a depressão na esquizofrenia tem impacto considerável na qualidade de vida do paciente. Sabe-se que, com seu tratamento correto, a morbimortalidade associada a ela seria reduzida em torno de $70 \% 22$.

O objetivo deste artigo foi estabelecer a prevalência pontual de depressão na esquizofrenia investigando os fatores associados e sua relação com a qualidade de vida.

\section{Método}

\section{Sujeitos}

Pacientes em acompanhamento ambulatorial foram recrutados de um Serviço de Referência em Saúde Mental de Belo Horizonte, Minas Gerais, Brasil. Os critérios de elegibilidade foram: diagnóstico confirmado de esquizofrenia (CID10) com evolução mínima de um ano, ter idade entre 18 e 55 anos, não apresentar transtorno neurológico, retardo mental ou história de dependência de substância química, além de apre- sentar quadro clínico estável nas três semanas precedentes à entrevista, indicados por ausência de hospitalização, depoimento de familiares e equipe do serviço.

\section{Procedimento}

Foi conduzido um estudo de delineamento transversal, em que os pacientes foram convidados a participar por meio de telefonema, convite pessoal na ocasião das consultas ou telegrama. Informações sócio-demográficas e clínicas foram coletadas. A qualidade de vida do paciente foi avaliada por meio da escala QLS-BR (Quality of Life Scale - versão para o Brasil) 23,24 e a depressão pela escala ECDE 14,20. Para evitar o viés de informação, todos os pacientes foram avaliados por dois entrevistadores, um primeiro aplicava o questionário com informações clínicas e sócio-demográficas e a escala QLS-BR. O segundo entrevistador, sem nenhuma informação da entrevista anterior, aplicava a escala ECDE de depressão.

As entrevistas foram conduzidas por um total de cinco entrevistadores, previamente treinados para o uso dos instrumentos (concordância $\geq$ $80 \%)$. Para garantir a reprodutibilidade das observações entre os entrevistadores, durante todo percurso da pesquisa, foi conduzido um estudo de confiabilidade interavaliador em uma subamostra. Dessa forma, 20 pacientes $(13,3 \%)$ foram avaliados simultaneamente por dois entrevistadores, em uma mesma entrevista, aplicando de forma independente as duas escalas. $\mathrm{O}$ pesquisador principal deste estudo foi considerado a referência neste procedimento.

Foi também conduzido um estudo de confiabilidade do diagnóstico de esquizofrenia com base em um sorteio aleatório de $10 \%$ dos pacientes entrevistados. Foi elaborada a história clínica do paciente por meio de informações do prontuário e enviada a um psiquiatra externo, para avaliação do diagnóstico de forma independente.

\section{Instrumentos}

Foi utilizado um questionário contendo informações sócio-demográficas e clínicas, tais como: sexo, idade, estado civil, renda, escolaridade, diagnóstico, duração da doença, número e classe de medicamentos, reação adversa, atividade ocupacional e satisfação com a saúde.

A depressão foi avaliada pela escala de depressão na esquizofrenia (ECDE) 14,20 composta por nove itens, inicialmente derivados do exame do estado psíquico 25 e da escala Hamilton para depressão 26. Ela permite avaliar a presença de 
depressão mensurando a gravidade da sintomatologia, discriminando os sintomas depressivos dos sintomas negativos e extrapiramidais 27 . Para cada item, o sintoma é pontuado em ausente, leve, moderado ou grave. A amplitude dos escores varia de 0 a 27, quanto maior o escore, maior a gravidade do quadro depressivo. A ECDE foi validada para o contexto brasileiro, apresentando boas características de validade e confiabilidade 14,20

A qualidade de vida foi avaliada por meio da escala QLS-BR, desenvolvida especificamente para pacientes com esquizofrenia 28 e validada para o Brasil, tendo apresentado validade e confiabilidade adequadas 23,24. Possui um total de três domínios: (1) domínio social, (2) domínio ocupacional e (3) domínio intrapsíquico e relações interpessoais. A qualidade de vida global corresponde à média dos escores nos 21 itens da escala. O instrumento inclui informações sobre sintomatologia e funcionamento do paciente nas três semanas precedentes à entrevista, sendo pontuado em uma escala de sete pontos, em que os maiores escores refletem uma melhor qualidade de vida.

\section{Análise estatística}

A prevalência de depressão maior foi determinada usando-se o ponto de corte do escore $\geq 6$ na ECDE, valor apropriado para o melhor contraponto entre sensibilidade e especificidade 14,20. Dois grupos foram formados: pacientes com depressão maior (escore $\geq 6$ ) e pacientes sem depressão maior (escore $<6$ ). Comparações das características clínicas, sócio-demográficas e dos escores de qualidade de vida foram feitas entre os grupos usando-se o teste de qui-quadrado de Pearson ou o teste não-paramétrico de MannWhitney. Quando apropriado, foi estimado o odds ratio (OR).

Foi também conduzida uma análise multivariada por meio da árvore de decisão utilizandose o algoritmo CART, abreviação de Classification and Regression Tree. Trata-se de um método desenvolvido por Breiman et al. ${ }^{29}$, tendo como objetivo estabelecer uma relação entre variáveis preditoras e uma única variável resposta. A árvore é obtida por meio de sucessivas divisões binárias no conjunto de dados, de forma a tornar os subconjuntos cada vez mais homogêneos em relação à variável reposta.

Para as análises univariadas e multivariadas, a variável resposta foi a presença (escore $\geq 6$ ) ou ausência de depressão maior (escore $<6$ ) e as variáveis explicativas foram de natureza sóciodemográficas, clínicas e de qualidade de vida, incluindo o escore global e os três domínios da
QLS-BR em sua forma dimensional. Na análise multivariada, por meio da árvore de classificação, após a construção do nó raiz, foi incluída a estratificação da árvore pelos escores de qualidade de vida.

Para a análise utilizando-se o CART, todas as variáveis explicativas entraram inicialmente na árvore de regressão, com exceção da classe dos medicamentos, devido a sua alta correlação com a própria depressão. A importância relativa do nó foi avaliada pela diminuição na heterogeneidade do galho, quando comparado com o nó raiz, fornecido pela medida denominada improvement, utilizando-se o índice Gini de 0,001 29. O ajuste do modelo final foi avaliado por meio da estimativa de risco, que identifica casos que foram incorretamente classificados. Ou seja, ela avalia em que medida o método corretamente prediz os resultados, analisando a diferença entre o valor esperado e o observado pelo modelo.

Os dados foram também analisados por meio de uma regressão logística binária, com o principal objetivo de validar os resultados da árvore de decisão. Seu procedimento consistiu em eliminar individualmente as variáveis. Foi considerado como critério para permanência no modelo final se variáveis com valor $\mathrm{p} \leq 0,05$ e intervalo de confiança de 95\% (IC95\%) para a OR 30 . Foram utilizados os programas SPSS 11.5 (SPSS Inc., Chicago, Estados Unidos) e o Answertree 3.0 (STATCON, Witzenhausen, Alemanha).

O projeto foi aprovado pelo comitê de ética em pesquisa da Universidade Federal de Minas Gerais (UFMG), sob parecer 035/01.

\section{Resultados}

Foram encontrados no serviço 329 pacientes elegíveis para o estudo. Destes, 150 foram entrevistados. Os principais motivos relacionados à não-participação dos pacientes foram: não localização no endereço do prontuário (22\%); em processo de vinculação com outros serviços (8\%); não preenchimento dos critérios de inclusão no momento da entrevista ( $12 \%$ ) ou com dificuldades em estabelecer contato (10\%). Não houve diferença estatisticamente significativa entre pacientes elegíveis e pacientes entrevistados quanto a sexo, idade, diagnóstico e local de residência $(p>0,05)$.

A prevalência de depressão maior diagnosticada pela ECDE no grupo foi de 56\% (IC95\%: 47,7-64,0), totalizando 84 pacientes. Os resultados da análise univariada para a presença de depressão maior e a distribuição dos pacientes nas características sócio-demográficas e clínicas estão apresentados nas Tabelas 1 e 2. 
Para as características sócio-demográficas, observou-se diferença significativa entre os grupos apenas para a realização de atividades no lar ( $p=0,01)$. Os pacientes com depressão maior apresentaram maior chance de não relatarem atividades no lar (OR = 2,80; IC95\%: 1,24-6,30). Nenhuma outra variável foi significativa para a presença de transtorno depressivo na análise (Tabela 1).
Dentre as características clínicas, um número maior de variáveis esteve associado à depressão maior (Tabela 2). Pacientes com depressão maior tiveram maior chance de apresentar sintomas $(\mathrm{OR}=5,26$; IC95\%: 2,50-11,11), cerca de $70 \%$ deles relataram alguma sintomatologia nas três últimas semanas. Os principais sintomas referidos foram: tristeza, alucinações, cefaléia, impregnação neuroléptica, insônia, dor no corpo e

Tabela 1

Distribuição dos pacientes nas variáveis sócio-demográficas e resultado da regressão logística univariada para a presença de depressão maior *.

\begin{tabular}{|c|c|c|c|c|}
\hline Variáveis sócio-demográficas & $\begin{array}{l}\text { Percentual de pacientes com } \\
\text { depressão maior }(n=84)\end{array}$ & $\begin{array}{l}\text { Percentual de pacientes sem } \\
\text { depressão maior }(n=66)\end{array}$ & OR & Valor $\mathrm{p}$ \\
\hline Sexo & & & & 0,14 \\
\hline Feminino & 32,1 & 43,9 & 1,00 & \\
\hline Masculino & 67,9 & 56,1 & 1,66 & \\
\hline Idade (anos) & & & & 0,47 \\
\hline$>33$ & 44,0 & 50,0 & 1,00 & \\
\hline$\leq 33$ & 56,0 & 50,0 & 1,27 & \\
\hline Escolaridade (anos) & & & & 0,39 \\
\hline$\geq 4$ & 29,8 & 36,4 & 1,00 & \\
\hline$<4$ & 70,2 & 63,6 & 1,35 & \\
\hline Estado civil & & & & 0,35 \\
\hline Casado & 14,3 & 20,0 & 1,00 & \\
\hline Solteiro & 85,7 & 80,0 & 1,49 & \\
\hline Renda familiar (salários mínimos) & & & & 0,82 \\
\hline$>2$ & 68,8 & 70,5 & 1,00 & \\
\hline$\leq 2$ & 31,3 & 29,5 & 1,08 & \\
\hline Número de pessoas na residência & & & & 0,81 \\
\hline$\leq 4$ & 48,8 & 50,8 & 1,00 & \\
\hline$>4$ & 51,2 & 49,2 & 1,08 & \\
\hline Com quem mora & & & & 0,77 \\
\hline Esposa(o) & 9,5 & 12,1 & 1,00 & \\
\hline Pais & 61,9 & 63,6 & 1,23 & \\
\hline Outros & 28,6 & 24,2 & 1,21 & \\
\hline Trabalha atualmente & & & & 0,63 \\
\hline Sim & 8,3 & 10,6 & 1,00 & \\
\hline Não & 91,7 & 89,4 & 1,30 & \\
\hline Recebe auxílio doença & & & & 0,13 \\
\hline Não & 45,2 & 57,6 & 1,00 & \\
\hline $\operatorname{Sim}$ & 54,8 & 42,4 & 1,64 & \\
\hline Faz atividades no lar & & & & 0,01 \\
\hline Sim & 66,7 & 84,8 & 1,00 & \\
\hline Não & 33,3 & 15,2 & 2,80 & \\
\hline Participação em oficinas & & & & 0,22 \\
\hline Não & 78,6 & 86,4 & 1,00 & \\
\hline Sim & 21,4 & 13,6 & 1,72 & \\
\hline Encaminhamento outro serviço & & & & 0,86 \\
\hline Sim & 60,7 & 62,1 & 1,00 & \\
\hline Não & 39,3 & 37,9 & 1,06 & \\
\hline
\end{tabular}

* Foi utilizado o ponto de corte de escore $\geq 6$ na Escala Calgary de Depressão na Esquizofrenia (ECDE) como marcador de depressão maior. 
delírios. Pacientes com depressão maior também apresentaram maior chance de usar três ou mais medicamentos (OR = 1,55; IC95\%: 1,16-2,06) e de relatarem reação adversa à medicação $(\mathrm{OR}=$ 1,85; IC95\%: 0,96-3,57), ainda que limítrofe ( $\mathrm{p}=$ 0,06). Quanto à classe da medicação, relataram mais freqüentemente o uso de benzodiazepíni$\cos (\mathrm{OR}=3,57$; IC95\%: 1,69-7,69), antidepressivo ( $\mathrm{p}<0,01)$ e neurolépticos de depósito $(\mathrm{OR}=5,88$; IC95\%: 1,61-20,00).

Na Tabela 3, encontra-se a distribuição dos sintomas depressivos para os dois grupos de pacientes nos itens da ECDE. Observa-se diferença estatisticamente significante para todos os itens $(\mathrm{p}<0,01)$. No grupo de pacientes com depressão maior, 68 deles (80,9\%) apresentaram humor deprimido e o entrevistador observou depressão em 71 (84,5\%). O item suicídio foi pontuado como presente em 62 pacientes com depressão maior, dentre estes, 26 (42\%) relataram pensamentos ou planos, enquanto 36 pacientes (58\%) chegaram à tentativa de suicídio propriamente dita em algum momento de suas vidas. Para pacientes sem depressão maior, o principal sintoma detectado pela ECDE foi a depressão matutina, observada em 23 pacientes deste grupo (34,8\%). Diferença significativa entre o escore médio da ECDE para os dois grupos foi observada, com média de 10,9 para pacientes com depressão maior e 2,7 para aqueles sem depressão maior ( $\mathrm{p}<0,01)$.

A distribuição dos escores de qualidade de vida está apresentada na Tabela 4. No domínio social da QLS-BR, apenas um item, representado pelas relações familiares, foi significativo, apontando diferença entre pacientes com e sem depressão maior $(\mathrm{p}<0,01)$. Por outro lado, o domínio ocupacional foi significativo, incluindo quatro de seus cinco itens: nível de realização ( $\mathrm{p}<$ $0,01)$; subemprego $(p=0,06)$ ainda que limítrofe; satisfação ocupacional $(\mathrm{p}<0,01)$ e utilização do

Distribuição dos pacientes nas variáveis clínicas e resultado da regressão logística univariada para a presença de depressão maior *.

\begin{tabular}{|c|c|c|c|c|}
\hline Variáveis clínicas & $\begin{array}{l}\text { Percentual de pacientes com } \\
\text { depressão maior }(n=84)\end{array}$ & $\begin{array}{l}\text { Percentual de pacientes sem } \\
\text { depressão maior }(n=66)\end{array}$ & OR & Valor $\mathrm{p}$ \\
\hline Diagnóstico & & & & 0,84 \\
\hline Esquizofrenia paranóide & 77,4 & 78,8 & 1,00 & \\
\hline Outros subtipos & 22,6 & 21,2 & 1,09 & \\
\hline Duração da doença (anos) & & & & 0,69 \\
\hline$>5$ & 56,6 & 60,0 & 1,00 & \\
\hline$\leq 5$ & 43,4 & 40,0 & 1,15 & \\
\hline História de internação psiquiátrica & & & & 0,91 \\
\hline Não & 34,5 & 35,4 & 1,00 & \\
\hline $\operatorname{Sim}$ & 65,5 & 64,6 & 1,04 & \\
\hline Satisfação com a saúde & & & & 0,10 \\
\hline Sim & 85,7 & 93,9 & 1,00 & \\
\hline Não & 14,3 & 6,1 & 2,58 & \\
\hline Presença de sintomas da doença & & & & $<0,01$ \\
\hline Não & 33,3 & 72,3 & 1,00 & \\
\hline Sim & 66,7 & 27,7 & 5,26 & \\
\hline Tratamento atual & & & & 0,34 \\
\hline Não faz tratamento & 2,4 & 7,7 & 1,00 & \\
\hline Apenas usa medicamento & 3,6 & 4,6 & 2,50 & \\
\hline Tratamento ambulatorial & 94,0 & 87,7 & 3,46 & \\
\hline Uso do medicamento & & & & 0,10 \\
\hline Não usa & 2,4 & 7,6 & 1,00 & \\
\hline Uso regular & 78,6 & 83,3 & 3,00 & \\
\hline Uso irregular & 19,0 & 9,1 & 6,67 & \\
\hline Número de medicamentos & & & & $<0,01$ \\
\hline $0-2$ & 28,6 & 53,8 & 1,00 & \\
\hline$\geq 3$ & 71,4 & 46,2 & 1,55 & \\
\hline
\end{tabular}

(continua) 


\begin{tabular}{|c|c|c|c|c|}
\hline Variáveis clínicas & $\begin{array}{l}\text { Percentual de pacientes com } \\
\text { depressão maior }(n=84)\end{array}$ & $\begin{array}{l}\text { Percentual de pacientes sem } \\
\text { depressão maior }(n=66)\end{array}$ & OR & Valor $\mathrm{p}$ \\
\hline Relato de reação adversa & & & & 0,06 \\
\hline Não & 39,3 & 54,5 & 1,00 & \\
\hline Sim & 60,7 & 45,5 & 1,85 & \\
\hline Atividade delirante na entrevista & & & & 0,11 \\
\hline Não & 79,8 & 89,4 & 1,00 & \\
\hline Sim & 20,2 & 10,6 & 2,12 & \\
\hline \multicolumn{5}{|l|}{ Classe de medicamentos } \\
\hline Estabilizador de humor & & & & 0,85 \\
\hline Sim & 9,3 & 10,3 & 1,00 & \\
\hline Não & 90,7 & 89,7 & 1,21 & \\
\hline Anticolinérgico & & & & 0,80 \\
\hline Não & 48,6 & 50,8 & 1,00 & \\
\hline Sim & 51,4 & 49,2 & 1,08 & \\
\hline Benzodiazepínicos & & & & $<0,01$ \\
\hline Não & 46,7 & 75,9 & 1,00 & \\
\hline Sim & 53,3 & 24,1 & 3,57 & \\
\hline Antidepressivo & & & & $<0,01$ \\
\hline Sim & 10,7 & - & 1,00 & \\
\hline Não & 89,3 & 100,0 & - & \\
\hline Neuroléptico típico & & & & 0,25 \\
\hline Sim & 82,7 & 89,7 & 1,00 & \\
\hline Não & 17,3 & 10,3 & 1,82 & \\
\hline Neuroléptico atípico & & & & 0,58 \\
\hline Não & 82,7 & 86,2 & 1,00 & \\
\hline $\operatorname{Sim}$ & 17,3 & 13,8 & 1,32 & \\
\hline Neuroléptico típico de depósito & & & & $<0,01$ \\
\hline Não & 76,0 & 94,8 & 1,00 & \\
\hline $\operatorname{Sim}$ & 24,0 & 5,28 & 5,88 & \\
\hline
\end{tabular}

* Foi utilizado o ponto de corte de escore $\geq 6$ na Escala Calgary de Depressão na Esquizofrenia (ECDE) como marcador de depressão maior.

Tabela 3

Distribuição dos sintomas depressivos identificados pela Escala Calgary de Depressão na Esquizofrenia (ECDE) e teste de comparação entre grupos *.

\begin{tabular}{|c|c|c|c|c|}
\hline \multirow[t]{2}{*}{ Sintomas da ECDE } & \multicolumn{2}{|c|}{$\begin{array}{l}\text { Pacientes com depressão maior } \\
\qquad(\mathrm{n}=84)\end{array}$} & \multicolumn{2}{|c|}{$\begin{array}{l}\text { Pacientes sem depressão maior } \\
\qquad(n=66)\end{array}$} \\
\hline & $\mathrm{n}$ & $\%$ ** & $n$ & $\%$ \\
\hline 1. Humor deprimido & 68 & 80,9 & 22 & 33,3 \\
\hline 2. Desesperança & 57 & 67,9 & 10 & 15,1 \\
\hline 3. Autodepreciação & 56 & 66,7 & 12 & 18,2 \\
\hline 4. Idéias de referência de culpa & 29 & 34,5 & 13 & 19,7 \\
\hline 5. Culpa patológica & 52 & 61,9 & 19 & 28,8 \\
\hline 6. Depressão matutina & 51 & 60,7 & 23 & 34,8 \\
\hline 7. Despertar precoce & 50 & 59,5 & 17 & 25,7 \\
\hline 8. Suicídio & 62 & 73,8 & 13 & 19,7 \\
\hline 9. Depressão observada & 71 & 84,5 & 10 & 15,1 \\
\hline Média (desvio padrão) da ECDE & \multicolumn{2}{|c|}{$10,98(4,79)$} & \multicolumn{2}{|c|}{$2,71(1,88)$} \\
\hline Amplitude & \multicolumn{2}{|c|}{$6-24$} & \multicolumn{2}{|c|}{$0-5$} \\
\hline
\end{tabular}

* Comparação de proporção: todos os itens $p<0,01$;

** Percentual obtida considerando os 66 pacientes sem depressão e 84 com depressão maior. 
Distribuição dos escores de qualidade de vida na escala QLS-BR (Quality of Life Scale - versão para o Brasil) de acordo com a presença e ausência de depressão * e resultado do teste de Mann-Whitney para a comparação entre os grupos.

\begin{tabular}{|c|c|c|c|}
\hline Domínios, itens e escala global & $\begin{array}{l}\text { Mediana dos pacientes com } \\
\text { depressão maior }(n=84)\end{array}$ & $\begin{array}{c}\text { Mediana dos pacientes sem } \\
\text { depressão maior }(n=66)\end{array}$ & Valor $\mathrm{p}$ \\
\hline Domínio social & 2,14 & 2,42 & 0,15 \\
\hline 1. Relações familiares & 3,33 & 4,00 & $<0,01$ \\
\hline 2. Relações íntimas & 1,00 & 1,50 & 0,50 \\
\hline 3. Amizades ativas & 2,00 & 2,00 & 0,57 \\
\hline 4. Atividade social & 1,00 & 2,00 & 0,28 \\
\hline 5. Rede social & 2,00 & 2,00 & 0,30 \\
\hline 6. Iniciativa social & 2,00 & 2,00 & 0,51 \\
\hline 7. Afastamento social & 2,00 & 2,00 & 0,17 \\
\hline Domínio ocupacional & 2,80 & 3,77 & $<0,01$ \\
\hline 9. Funcionamento ocupacional & 3,00 & 4,00 & 0,07 \\
\hline 10. Nível de realização & 3,00 & 4,00 & $<0,01$ \\
\hline 11. Subemprego & 3,00 & 4,00 & 0,06 \\
\hline 12. Satisfação ocupacional & 2,50 & 3,37 & $<0,01$ \\
\hline 17. Utilização do tempo & 2,00 & 4,00 & $<0,01$ \\
\hline Domínio intrapsíquico e relações interpessoais & 2,85 & 3,07 & 0,19 \\
\hline 8. Relações afetivo-sexuais & 1,00 & 1,00 & 0,38 \\
\hline 13. Sentido de objetivos de vida & 3,00 & 3,00 & 0,50 \\
\hline 15. Curiosidade & 3,00 & 2,00 & 0,71 \\
\hline 18. Objetos comuns & 4,00 & 4,00 & 0,65 \\
\hline 19. Atividades comuns & 2,00 & 3,00 & 0,16 \\
\hline 20. Empatia & 4,00 & 4,00 & 0,53 \\
\hline 21. Interação na entrevista & 3,00 & 3,00 & 0,21 \\
\hline Qualidade de vida global (21 itens) & 2,50 & 2,93 & 0,01 \\
\hline 14. Motivação & 2,00 & 4,00 & $<0,01$ \\
\hline 16. Anedonia & 2,00 & 4,00 & $<0,01$ \\
\hline
\end{tabular}

* Foi utilizado o ponto de corte de escore $\geq 6$ na Escala Calgary de Depressão na Esquizofrenia (ECDE) como marcador de depressão maior.

tempo $(\mathrm{p}<0,01)$. O domínio referente às funções intrapsíquicas e relações interpessoais não mostrou diferença entre os grupos. A escala global, que corresponde à média dos 21 itens, apresentou diferença significativa entre pacientes com e sem depressão maior $(\mathrm{p}<0,01)$, além dos itens motivação $(\mathrm{p}<0,01)$ e anedonia $(\mathrm{p}<0,01)$.

Na Tabela 5, estão apresentados os resultados da análise univariada do escore dimensional da QLS-BR na presença de depressão maior. O escore global de qualidade de vida $(p=0,01)$ e $o$ domínio ocupacional $(\mathrm{p}<0,01)$ estiveram associados com transtorno depressivo, sugerindo que a cada diminuição de um ponto no escore global de qualidade de vida tem-se 1,5 mais chance do paciente apresentar depressão maior $(\mathrm{OR}=1,51$; IC95\%: 1,09-2,13), enquanto esta mesma chance para o domínio ocupacional foi de $1,3(\mathrm{OR}=1,37$; IC95\%: 1,09-1,72). O domínio social, das funções intrapsíquicas e relações interpessoais não foram significativos.
Tabela 5

Resultado da análise univariada do escore dimensional de qualidade de vida na escala QLSBR (Quality of Life Scale - versão para o Brasil) para pacientes com e sem depressão maior.

\begin{tabular}{lccc}
\hline Escores de qualidade de vida & OR & IC95\% & Valor $\mathbf{p}$ \\
\hline Escala global & 1,51 & $1,09-2,13$ & 0,01 \\
Domínio social & 1,23 & $0,95-1,61$ & 0,11 \\
Domínio ocupacional & 1,37 & $1,09-1,72$ & $<0,01$ \\
Domínio intrapsíquico e interpessoal & 1,27 & $0,92-1,72$ & 0,16 \\
\hline
\end{tabular}

A Figura 1 apresenta os resultados da análise multivariada pela árvore de classificação. Observa-se o nó raiz com a amostra total dos pacientes, dos quais $56 \%$ deles apresentaram depressão maior. O escore de qualidade de vida discriminou bem esse grupo de pacientes, diminuindo 
Figura 1

Resultado da árvore de classificação pelo algoritmo CART (Classification and Regression Tree) para o escore de depressão na Escala Calgary de Depressão na Esquizofrenia (ECDE) estratificado pelo escore de qualidade de vida na QLS-BR (Quality of Life Scale - versão para o Brasil) $(n=150)$.

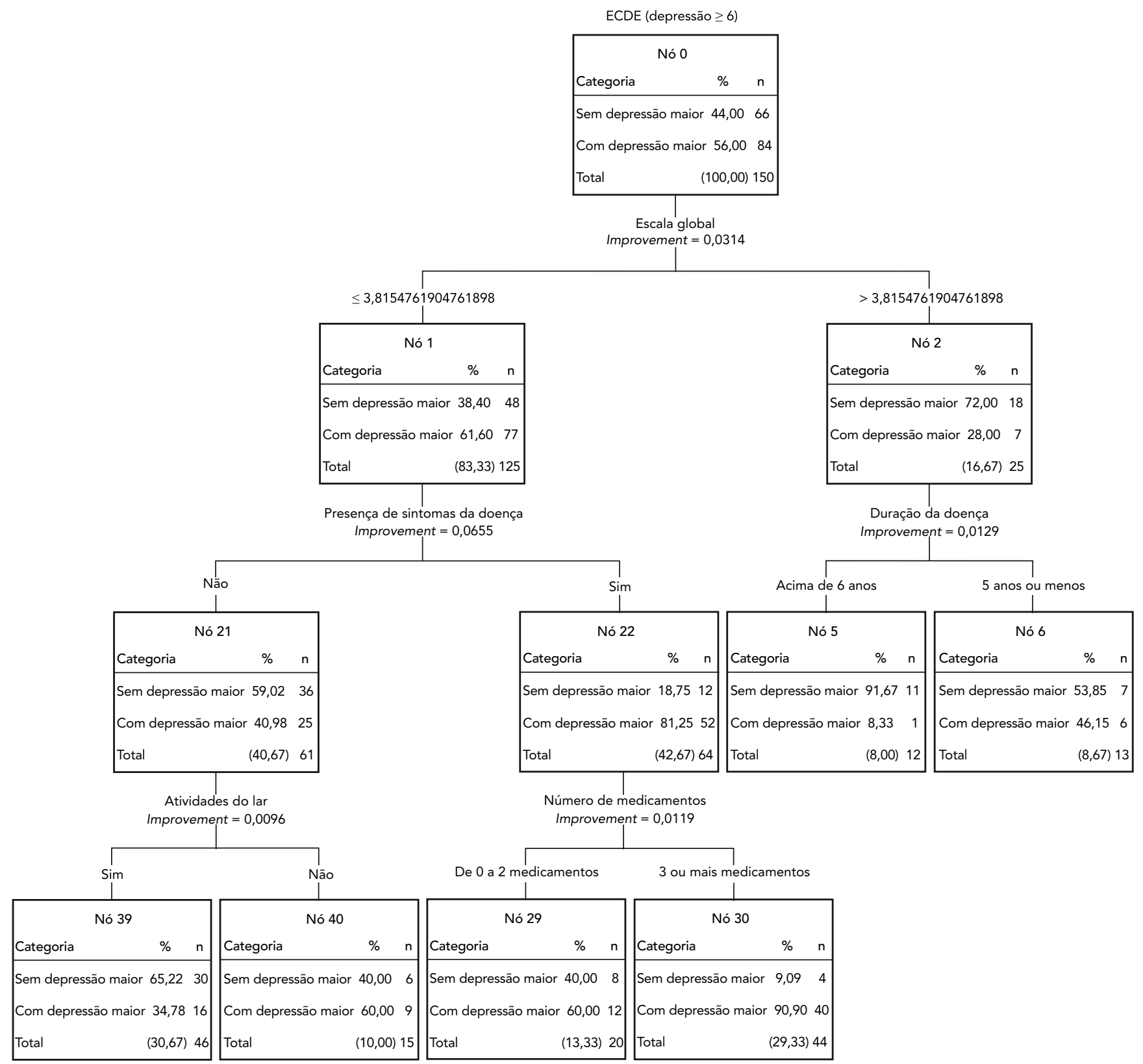

a heterogeneidade do nó (improvement $=0,03$ ). Para aqueles pacientes com escore de qualidade de vida maior que 3,81 e duração da doença acima de seis anos, apenas $8,3 \%$ deles apresentaram depressão maior, enquanto, aqueles pacien- tes com escore de qualidade de vida maior que 3,81 , mas com duração da doença menor que seis anos, $46 \%$ deles apresentaram maior gravidade da sintomatologia depressiva, sugerindo depressão maior. 
Por outro lado, os pacientes com escore de qualidade de vida menor ou igual a 3,81, que relataram presença de sintomas da doença e usavam mais de três medicamentos, 90\% deles apresentaram depressão maior comparados com $60 \%$ para aqueles usando até dois medicamentos. Ainda com níveis similares nos escores qualidade de vida, pacientes que não relataram sintomas da doença e que não desenvolviam atividade do lar, $60 \%$ apresentaram depressão maior; para o mesmo grupo que realizava alguma atividade no lar, 35\% apresentaram depressão maior. O ajuste da árvore de classificação indicou que $73 \%$ dos pacientes foram corretamente classificados (risco = 0,27 ), ou seja, com risco de $27 \%$ de classificação incorreta.

A regressão logística multivariada apresentou resultados similares aos encontrados na árvore de classificação, indicando as seguintes variáveis associadas à presença de transtorno depressivo maior: relato de sintomas ( $\mathrm{OR}=5,50$; IC95\%: 2,56-12,50), ausência de atividades do lar (OR = 2,98; IC954\%: 1,15-7,66) e uso de três ou mais medicamentos (OR = 2,71; IC95\%: 1,25-5,90).

A confiabilidade diagnóstica por meio da avaliação do psiquiatra independente foi de $95 \%$. Apenas para um paciente não se obteve dados suficientes da história clínica no prontuário para confirmação do diagnóstico de esquizofrenia. $\mathrm{O}$ coeficiente kappa ponderado foi superior a $90 \%$ indicando excelente confiabilidade interavaliadores para a aplicação dos instrumentos na condução do estudo.

\section{Discussão}

Neste estudo foi encontrada alta prevalência de depressão em pacientes ambulatoriais com diagnóstico de esquizofrenia e quadro estável. Indicadores clínicos e ocupacionais, como relato de sintomatologia, maior duração da doença, uso de maior número de medicamentos e ausência de atividades no lar, mostraram-se associadas com a maior gravidade da sintomatologia depressiva nestes pacientes.

Foi evidenciada importante relação entre depressão e baixa qualidade de vida, principalmente no domínio ocupacional, em que pacientes com depressão mostraram-se menos realizados, insatisfeitos, subutilizados no tempo e em sua capacidade para o trabalho. Também apresentaram-se com baixos escores de qualidade de vida nas relações familiares, menos motivados e com baixa capacidade de sentir prazer na vida, quando comparados com pacientes sem depressão.

A prevalência da depressão encontrada neste estudo está dentro da amplitude relatada por uma revisão sistemática sobre o tema 11 , embora estudos conduzidos no Brasil utilizando diferentes abordagens metodológicas apresentem taxas inferiores às encontradas nesta investigação 13,14,15. Entretanto, sabe-se que sintomas depressivos podem estar presentes em qualquer fase da esquizofrenia, seja como pródomo de um novo episódio psicótico, na fase aguda da doença, no período pós-psicótico ou na fase residual 15,31. Nesta investigação, embora seja difícil a identificação da fase da doença em que os sintomas depressivos estavam ocorrendo, foi encontrado um grande número de pacientes apresentando sintomatologia depressiva, ressaltando a importância desta morbidade e seu considerável impacto na gravidade do quadro clínico e na qualidade de vida do paciente.

Analisando-se a distribuição dos escores da escala de depressão aqui utilizada (ECDE) observou-se que, no grupo de pacientes com depressão maior a presença de humor deprimido, tentativa de suicídio, desesperança, autodepreciação e depressão matutina foram freqüentes e coerentes com outros resultados da literatura 13,14,15,27,32. Chama a atenção o grande percentual de pacientes com tentativa de suicídio encontrado neste estudo, $58 \%$ da amostra investigada. Esse achado é relevante 33,34 tendo em vista a morbimortalidade nessa população associada a esta causa 33,34,35,36,37. Apesar da escassez de estudos sobre tentativas de suicídio em pacientes com esquizofrenia, as taxas descritas variam de $18 \%$ a $55 \%$, dependendo da natureza do estudo 38 . Tem sido relatado que tentativas de suicídio são observadas com maior freqüência nos primeiros anos da doença ou durante os períodos de exacerbação da sintomatologia 39 .

Em relação ao suicídio propriamente dito, estudos epidemiológicos relatam que de $2 \%$ a $13 \%$ de todos os pacientes com esquizofrenia cometem suicídio, risco $20 \%$ maior que o da população geral 40. Estão associadas a esse episódio, além de ser do sexo masculino e idade mais jovem, outros fatores, tais como: ser solteiro, vivenciar isolamento social, estar desempregado, ter expectativa de bom prognóstico da doença, ter estado deprimido no passado e no presente, além do próprio relato de tentativas de suicídio 37,40 .

Dentre as variáveis sócio-demográficas, apenas ausência de atividades no lar foi associada com depressão na análise multivariada. Soma-se a esse resultado, para os pacientes com maior sintomatologia depressiva, o achado de baixa qualidade de vida no domínio ocupacional, além de baixos escores para os itens motivação e anedonia. Uma possível explicação para o fato do domínio ocupacional ter se destacado diante dos demais, se deve ao fato de a escala QLS-BR privi- 
legiar atividades de vida cotidiana, incluindo realização de atividades domésticas. Uma vez que os pacientes brasileiros não possuem outros vínculos sociais importantes, esse domínio propiciou a diferenciação entre pacientes com maior e menor gravidade da sintomatologia depressiva. Vale ressaltar que a participação em atividades ocupacionais é vista pelos pacientes como uma possibilidade de distração dos sintomas, contribuindo para um melhor estado mental. Tal informação poderia ajudar a compreender a associação entre sintomas depressivos e baixo escore de qualidade de vida no domínio ocupacional da escala QLS-BR.

Investigações anteriores utilizando a escala QLS-BR apontaram para uma associação entre transtorno de humor, avaliado pela Escala de Sintomas Positivos e Negativos (PANSS), e baixa qualidade de vida no domínio interpessoal e escore global ${ }^{41}$. Outros estudos mostraram uma mais baixa qualidade de vida na dimensão ocupacional principalmente para homens solteiros 42,43 . Embora seja relatado pior funcionamento ocupacional em pacientes com depressão 7,10, investigações comparando pacientes com e sem depressão não encontraram diferenças em relação às características sócio-demográficas 14,44.

Com relação às variáveis clínicas, a presença de sintomas esteve associada com a depressão, podendo ser apontada como indicador da própria doença, uma vez que os sintomas referidos pelos pacientes têm estreita relação com quadro depressivo 45,46. Na árvore de decisão, antes da estratificação pela qualidade de vida foi a variável que melhor discriminou os dois grupos de pacientes, mostrando distintas variáveis associadas com a depressão na presença ou ausência de sintomatologia (dados não mostrados). Após a estratificação pela qualidade de vida, permaneceu associada para aqueles pacientes com menores escores de qualidade de vida (escore $\leq 3,82$ ).

A duração da doença foi outro marcador clínico importante para os pacientes com melhores escores de qualidade de vida (escore $\geq 3,82$ ) na árvore de classificação, embora esta diferença não tenha sido evidenciada no modelo de regressão logística. Por limitações do delineamento do estudo, a associação entre maior duração da doença e maior sintomatologia depressiva não pode ser facilmente explicada. Podem ser levantadas algumas proposições. Dessa forma, uma menor duração da doença pode refletir no paciente o sentimento de perdas de capacidades anteriores, decorrente da doença ainda recente 12 . Outra possibilidade seria que os pacientes com maior duração da doença teriam mais chances de estar com o quadro clínico estável, seja pelo maior tempo de acompanhamento e intervenção do serviço ou pela própria história natural da depressão na esquizofrenia 11,14,39.

Diferenças significativas entre pacientes com e sem episódio depressivo em relação à duração da doença não foi evidenciado em outros estudos 33. Apesar de apresentar-se com resultados contraditórios, em diferentes investigações ou com diferentes abordagens estatísticas, é importante considerar a relevância clínica da duração da doença na sintomatologia depressiva, em pacientes com esquizofrenia.

O relato do uso de maior número de medicamentos também esteve associado à presença de depressão maior. Cerca de $72 \%$ dos pacientes deprimidos usavam mais de três medicamentos. $\mathrm{Na}$ análise exploratória dos dados, considerando-se a combinação dos medicamentos, foi observado que pacientes deprimidos apresentaram maior variedade de combinações, somando-se ainda, maior presença de sintomatologia da doença. Essas informações podem sugerir uma estratégia de intervenção medicamentosa do serviço, baseada no relato do paciente sobre a presença de sintomatologia da doença, embora a depressão seja pouco reconhecida enquanto morbidade importante nesta população. O pequeno percentual de pacientes em uso de antidepressivo $(10,7 \%)$ reforça este achado de possível subdiagnóstico de depressão nesta população.

Em se tratando da intervenção medicamentosa para a depressão na esquizofrenia, sabe-se que a mesma está diretamente relacionada com o conhecimento da fase da doença e que, às vezes, não requer a utilização de antidepressivos $11,14,39$. Por outro lado, pacientes com esquizofrenia e quadro depressivo podem não estar sendo medicados de forma específica ou, quando isto é feito, o antidepressivo é prescrito em dosagem inferior à necessária 11,33,39.

A associação entre depressão e uso de antidepressivos, benzodiazepínicos e neurolépticos de depósito encontrada na análise univariada não pode ser subestimada. Estudos relatam uma associação entre medicação de depósito e depressão, sugerindo que estas drogas podem induzir à "depressão farmacogênica”, principalmente quando usada em dosagem maior do que a necessária 47 . Em nosso estudo, 95\% dos pacientes relataram o uso de medicação neuroléptica, dentre estes, $24 \%$ usavam medicação de depósito. Esse fato merece outras investigações uma vez que não foi coletada a informação sobre dosagem, em detrimento de dados controversos na literatura a respeito de sua associação com a depressão 48 .

Da mesma forma, os neurolépticos têm sido investigados quanto à possibilidade de atuarem como fatores coadjuvantes, facilitadores ou pre- 
cipitadores da síndrome depressiva pós-psicótica ${ }^{12}$. Investigações recentes apontam que seu uso pode estar associado com sintomas depressivos 49 .

Algumas considerações são importantes para a compreensão da alta prevalência de depressão encontrada nesta investigação. Assim como em outros estudos 15,46, utilizou-se como critério de inclusão pacientes clinicamente estáveis, como forma de evitar a mensuração de sintomas depressivos ocorrendo durante o episódio psicótico agudo. Por outro lado, o período de três semanas de estabilidade pode não ter sido suficiente para evitar a sobreposição da depressão com a recaída psicótica, ou talvez com sintomatologia positiva. Alguns estudos utilizam um período mínimo de dois meses 15,46. Outra explicação possível seria o instrumento usado para identificação da depressão maior, utilizando o ponto de corte com escore $\geq 6$ na ECDE 14. Esta categorização não incluiu a necessidade do humor deprimido enquanto critério para o diagnóstico de depressão na esquizofrenia 45 .

Com a finalidade de verificar a especificidade do critério diagnóstico de depressão nesta investigação, análises estatísticas foram conduzidas retirando os pacientes que não apresentaram humor deprimido na escala ECDE. Como esperado, a prevalência global caiu de $56 \%$ para $45 \%$, e as mesmas variáveis permaneceram associadas com a depressão, com maior magnitude da força da associação no modelo logístico. Embora seja importante considerar que o objetivo deste estudo não foi validar o diagnóstico de depressão, mas sim mensurá-la por meio da ECDE que foi validada para o Brasil, mostrando ser equivalente aos critérios diagnósticos do DSM-IV (Diagnostic and Statistical Manual of Mental Disorders - fourth edition) 14 .

Controvérsias na literatura a respeito da prevalência de depressão na esquizofrenia podem também ser resultantes de divergências metodológicas, tais como: várias definições da depressão, uso de diferentes critérios diagnósticos de esquizofrenia, avaliação dos pacientes em fases distintas da doença, além de diferentes períodos de estabilidade do quadro clínico 15.

De forma geral, as características clínicas associadas com maior sintomatologia depressiva encontradas nesta investigação podem indicar pacientes com quadro clínico mais grave de esquizofrenia e, portanto mais incapacitados, necessitando de tratamento mais intensivo. Esta perspectiva poderia contribuir para uma baixa qualidade de vida nos pacientes avaliados 18 .

O transtorno depressivo é hoje considerado um problema de saúde pública devido à sua alta prevalência e impacto psicossocial. Quando associado à esquizofrenia, seu impacto pode ser ainda maior. Pacientes deprimidos são freqüentemente mais assíduos aos serviços de saúde 18, apresentam diminuição da produtividade no trabalho com repercussões importantes na qualidade de vida, embora não sejam diagnosticados como tais 9 devido a dificuldades inerentes ao diagnóstico, somando-se a uma atenção dos profissionais, focada quase que exclusivamente no quadro psicótico 18 .

Este estudo estima a prevalência de depressão nos pacientes com diagnóstico de esquizofrenia e aponta sua importante associação com a qualidade de vida. Considerando este estreito relacionamento, investigações sobre esta temática se tornam prioritárias. A presença de depressão é vista como principal fator associado com pior qualidade de vida subjetiva em pacientes com esquizofrenia 10. É conhecido por meio de sua história natural que toda doença está associada com um prejuízo marcante na qualidade de vida do paciente. Dentro desse contexto, todo tratamento, se efetivo, deve necessariamente melhorar a qualidade de vida. Atualmente, medidas de qualidade de vida e de depressão, sob o ponto de vista do paciente, têm sido valorizadas na psiquiatria 8,10 . Apesar de existir pouco consenso científico de como os sintomas depressivos na esquizofrenia devem ser definidos e diagnosticados, é reconhecida a importância da avaliação da depressão do ponto de vista do paciente ${ }^{48}$, mesmo que mediada por um instrumento.

É importante considerar a limitação desta investigação enquanto estudo seccional. Nesse sentido, não é possível estabelecer uma relação de temporalidade entre depressão e baixa qualidade de vida, pois os dados podem também apontar para uma relação inversa. Da mesma forma, para uma generalização dos resultados, outras investigações deverão ser conduzidas, incluindo grandes amostras, com melhor definição da fase da esquizofrenia em que os sintomas depressivos estão ocorrendo. Além disso, deve ser incluído mais de um critério de avaliação da depressão, dado a dificuldade inerente deste diagnóstico nessa população, embora o resultado desta investigação seja de considerável interesse.

Do ponto de vista de intervenções, é importante considerar a alta prevalência de sintomatologia depressiva nos pacientes, associada à necessidade de melhor avaliação deste aspecto, dentro de uma proposta clínica, principalmente considerando a alta proporção de pacientes com tentativas de suicídio anteriores. É importante reconhecer e avaliar a necessidade da implementação de propostas terapêuticas, com intervenções voltadas para a inserção social, uma vez que pacientes deprimidos podem apresentar baixa 
qualidade de vida 10, incluindo pior funcionamento social, dificuldades de relacionamento e insatisfação com suporte social recebido 48 .

Estudos prospectivos apontam que uma melhor qualidade de vida na esquizofrenia esteve associada com a existência de suporte social capaz de responder efetivamente às necessidades dos pacientes 10 . Torna-se ainda importante, uma reavaliação clínica detalhada da fase da doença em que está ocorrendo a sintomatologia depressiva, informações úteis no manejo clínico e na escolha da melhor estratégia terapêutica 39 . Da mesma forma faz-se necessário o desenvolvimento de estudos de acompanhamento desses pacientes,

\section{Resumo}

O objetivo deste estudo foi mensurar a prevalência de depressão na esquizofrenia, investigando os fatores associados e sua relação com a qualidade de vida. Foi conduzido um estudo transversal com 150 pacientes ambulatoriais, a depressão foi mensurada pela Escala Calgary de Depressão na Esquizofrenia e a qualidade de vida por meio da escala Quality of Life Scale-Brasil. Foi encontrada uma prevalência de $56 \%$ de depressão maior. Pacientes com depressão maior apresentaram pior qualidade de vida na escala global e domínio ocupacional. A qualidade de vida global foi importante para a separação dos grupos na árvore de decisão. Em pacientes com pior qualidade de vida, presença de sintomas da doença, número de medicamentos e ausência de atividades no lar se associaram à depressão, enquanto para aqueles com melhor qualidade de vida, apenas duração da doença foi importante. Esta investigação sugere alta prevalência de depressão, além de mostrar sua repercussão na qualidade de vida. Recomenda-se a investigação da depressão associada à qualidade de vida no cuidado terapêutico destes pacientes.

Depressão; Esquizofrenia; Qualidade de Vida uma vez que vários autores relatam a depressão como um período prolongado, sem relação com o tempo de permanência na psicose 12 , com prejuízo cognitivo e deficitário 15,33.

Os resultados deste estudo têm implicações importantes para o tratamento de pacientes com esquizofrenia. Ele confirma que a depressão é comum nesse grupo de pacientes e aponta para uma estreita relação entre depressão e baixa qualidade de vida. Dentro do contexto da inclusão de indicadores de qualidade de vida na rotina dos serviços de saúde mental, faz-se necessário que a gravidade da sintomatologia depressiva na esquizofrenia seja também avaliada.

\section{Colaboradores}

C. S. Cardoso elaborou o projeto, coordenou os trabalhos, participou do estudo de campo, supervisionou a entrada, a análise e consistência dos dados e redigiu o manuscrito. W. T. Caiaffa participou no desenho do estudo, em todo o processo de campo, auxiliou na entrada e análise de dados. M. Bandeira participou também no desenho do estudo e acompanhou o trabalho de campo. J. O. P. Fonseca participou na assessoria clínica. A análise estatística foi acompanhada por A. L. Siqueira. J. T. Silva auxiliou na entrada e análise de dados. Todos os autores ajudaram na redação do artigo, aprovando sua versão final.

\section{Agradecimentos}

Este artigo é parte integrante da Tese de Doutorado no Programa de Pós-graduação em Saúde Pública da Universidade Federal de Minas Gerais. Os autores agradecem à Fundação de Amparo à Pesquisa do Estado de Minas Gerais (FAPEMIG) pelo financiamento, processo $\mathrm{n}^{\circ}$. CDS-301/02 e também ao Conselho Nacional de Desenvolvimento Científico e Tecnológico (CNPq) e Coordenação de Aperfeiçoamento de Pessoal de Nível Superior (CAPES) pelas bolsas aos pesquisadores: Waleska Teixeira Caiaffa, Jussara Teixeira da Silva e Clareci Silva Cardoso. Os autores agradecem ao psiquiatra Helian Nunes pela colaboração no estudo de confiabilidade diagnóstica. Agradecem também ao Grupo de Pesquisa em Epidemiologia (GPE) e ao Observatório de Saúde Urbana de Belo Horizonte, pela estrutura de trabalho. Um agradecimento especial ao Centro de Referência em Saúde Menta Pampulha (CERSAM Pampulha) de Belo Horizonte, a todos os pacientes e familiares pelo apoio na produção científica. 


\section{Referências}

1. Huppert JD, Weis KA, Lim R, Pratt S, Smith TE. Quality of life in schizophrenia: contributions of anxiety and depression. Schizophr Res 2001; 51:171-80.

2. Becker RE. Depression in schizophrenia. Hosp Community Psychiatry 1988; 39:1269-75.

3. Roy A. Risk factors for suicide in psychiatric patients. Arch Gen Psychiatry 1982; 39:1089-95.

4. Drake FE, Gates G, Cotton PG, Whitaker A. Suicide among schizophrenics: who is at risk? J Nerv Ment Dis 1984; 172:613-7.

5. Glazer W, Prusoff B, John K, Williams D. Depression and social adjustment among chronic schizophrenic outpatients. J Nerv Ment Dis 1981; 169:712-7.

6. Hillmmelhoch JM, Fuchs CS, May SJ, Symons BJ, Neil JF. When a schizoaffective diagnoses has meaning. J Nerv Ment Dis 1981; 169:277-82.

7. Eklund M, Hansson L, Bejerholm U. Relationships between satisfaction with occupational factors and health-related variables in schizophrenia outpatients. Soc Psychiatry Psychiatr Epidemiol 2001; 36:79-85.

8. Carpinello B, Lai GL, Pariant CM, Carta MG, Rudas M. Symptoms, standards of living and subjective quality of life: a comparative study of schizophrenic and depressed out-patient. Acta Psychiatr Scand 1997; 96:235-41.

9. Fleck MPA, Lima AFBS, Louzada S, Schestask G, Henriques A, Borges VR, et al. Associação entre sintomas depressivos e funcionamento social em cuidados primários à saúde. Rev Saúde Pública 2002; 36:431-8.

10. Reine G, Lançon C, Di Tucci S, Sapin C, Auquier P. Depression and subjective quality of life in chronic phase schizophrenic patients. Acta Psychiatr Scand 2003; 108:297-303.

11. Siris SG. Diagnosis of secondary depression in schizophrenia: implications for DSM-IV. Schizophr Bull 1991; 17:75-98.

12. McGlashan TH, Carpenter Jr. WT. Postpsychotic depression in schizophrenia. Arch Gen Psychiatry 1976; 33:231-9.

13. Bastos O. Contribuição ao estudo clínico da depressão pós-esquizofrênica [Tese de Doutorado]. Recife: Faculdade de Ciências Médicas, Universidade de Pernambuco; 1981.

14. Bressan RA, Chaves AC, Shirakawa I, Mari JJ. Validity study of the Brazilian version of the Calgary depression scale for schizophrenia. Schizophr Res 1998; 32:41-9.

15. Araújo FS, Petribú K, Bastos O. Characterization of depressive syndrome in schizophrenic outpatients. Rev Bras Psiquiatr 2002; 24:18-25.

16. Martin RL, Cloninger RC, Guze SB, Clayton PJ. Frequency and differential diagnosis of depressive syndromes in schizophrenia. J Clin Psychiatry 1985; 46:9-13.

17. Knights A, Hirsh SR. "Reveled" depression and drug treatment for schizophrenia. Arch Gen Psychiatry 1981; 38:806-11.
18. Conley RR, Ascher-Svanum H, Zhu B, Faries DE, Kinon BJ. The burden of depressive symptoms in the long-term treatment of patients with schizophrenia. Schizophr Res 2007; 90:186-97.

19. Green MF, Nuechterlein KH, Ventura J, Mintz J. The temporal relationship between depressive and psychotic symptoms in recent-onset schizophrenia. Am J Psychiatry 1990; 147:179-82.

20. Bressan RA, Chaves AC, Shirakawa I, Mari JJ.Versão brasileira da escala Calgary de depressão para esquizofrenia. Rev Psiq Clin 1998; 25:344-51.

21. Sullivan G, Wells KB, Leak B. Clinical factors associated with better quality of life in a seriously mentally ill population. Hosp Community Psychiatry 1992; 43 794-8.

22. Docherty JP. Barriers to the diagnosis of depression in primary care. J Clin Psychiatry 1997; 58:5-10.

23. Cardoso CS, Bandeira M, Caiaffa WT, Siqueira AL, Fonseca IK, Fonseca JOP. Qualidades psicométricas da escala de qualidade de vida para pacientes com esquizofrenia: Escala QLS-BR. J Bras Psiquiatr 2003; 52:211-22.

24. Cardoso CS. Adaptação transcultural para o Brasil de uma escala de qualidade de vida para pacientes com esquizofrenia: Escala QLS [Dissertação de Mestrado]. Belo Horizonte: Escola de Medicina, Universidade Federal de Minas Gerais; 2001.

25. Wing JK, Cooper JE, Sartorius N. The measurement and classification of psychiatric symptoms. London: Cambridge University Press; 1974.

26. Hamilton M. A rating scale for depression. J Neurol Neurosurg Psychiatry 1960; 23:56-62.

27. Addington D, Addington J, Matricka-Tyndale E. Specificity of the Calgary depression scale for schizophrenia. Schizophr Res 1994; 11:239-44.

28. Heinrichs DW, Hanlon TE, Carpenter WT. The quality of life scale: an instrument for rating the schizophrenic deficit syndrome. Schizophr Bull 1984; 10:388-98.

29. Breiman LJH, Friedman RA, Stone CJ. Classification and regression trees. Belmont: Chapman \& Hall; 1984.

30. Hosmer Jr. DW, Lemeshow S. Applied logistic regression. 2nd Ed. New York: John Wiley and Sons; 2000.

31. Bartels SJ, Drake RE. Depressive symptoms in schizophrenia: comprehensive differential diagnosis. Compr Psychiatry 1988; 29:467-83.

32. Becker RE, Colliver JA, Versulst SJ. Diagnoses of secondary depression in schizophrenia. J Clin Psychiatry 1985; 46:4-8.

33. Roy A, Mazonson A, Pickar D. Attempted suicide in chronic schizophrenia. Br J Psychiatry 1984; 144:303-6.

34. Addington D, Addington J. Attempted suicide and depression in schizophrenia. Acta Psychiatr Scand 1992; 85:288-91.

35. Araújo FS, Petribú K, Bastos O. Depressão na esquizofrenia. Rev Bras Psiquiatr 2002; 24:86-93.

36. Roy A. Depression attempted suicide, and suicide in patients with chronic schizophrenia. Psychiatric Clin North Am 1986; 9:193-206. 
37. Drake FE, Gattes C, Whitaker A. Suicide among schizophrenics: a review. Compr Psychiatry 1985; 26: $90-100$.

38. Roy A. Relationship between depression and suicide behavior in schizophrenia. In: DeLisi LE, editor. Depression in schizophrenia. Washington DC: American Psychiatric Press; 1990. p. 3-23.

39. Bressan RA, Chaves AC, Shirakwa I. Depressão na esquizofrenia. In: Shirakawa I, Chaves AC, Mari JJ, organizadores. O desafio da esquizofrenia. São Paulo: Lemos Editorial; 1998. p. 119-32.

40. Mortensen PB. Suicide among schizophrenic patients: occurrence and risk factors. Clin Neuropharmacol 1995; 18:1-8.

41. Tollefeson GD, Andersen SW. Should we consider mood disturbance in schizophrenia as an important determinant of quality of life? J Clin Psychiatry 1999; 60 Suppl 5:23-9.

42. Cardoso CS, Caiaffa WT, Bandeira M, Siqueira AL, Abreu MNS, Fonseca JOP. Factors associated with a lower quality of life in schizophrenia. Cad Saúde Pública 2005; 21:1338-40.

43. Cardoso CS, Caiaffa WT, Bandeira M, Siqueira AL, Abreu MNS, Fonseca JOP. Qualidade de vida e dimensão ocupacional na esquizofrenia: uma comparação por sexo. Cad Saúde Pública 2006; 22:1303-14
44. Lindenmayer JP, Bernstein-Hyman R, Grochowski S. Five-factor model of schizophrenia. Initial validation. J Nerv Ment Dis 1994; 182:631-8.

45. American Psychiatric Association. Diagnostic and statistical manual of mental disorders DSM IV. $4^{\text {th }}$ Ed. Washington DC: American Psychiatric Press, 1994.

46. Bressan RA, Chaves AC, Phowsky LS, Shirakawa I, Mari JJ. Depressive episodes in stable schizophrenia: critical evaluation of the DSM-IV and ICD-10 diagnostic criteria. Psychiatry Res 2003; 117:47-56.

47. De Alarcon R, Carney MW. Severe depressive mood changes following slow-release intramuscular fluphenazine injection. Br Med J 1969; 3:564-7.

48. Baynes D, Mulholland C, Cooper SJ, Montgonery RC, MacFlynn G, Lynch G, et al. Depressive symptoms in stable chronic schizophrenia: prevalence and relationship to psychopathology and treatment. Schizophr Res 2000; 45:47-56.

49. Bressan RA, Mulligan RS, Costa DC, Jones HM, Ell PJ, Kerwin RW, et al. Striatal D2 blockade and depression in schizophrenia - SPET findings. Biol Psychiatry 2000; 47(8 Suppl 1):S128.

Recebido em 08/Ago/2006

Versão final reapresentada em 13/Fev/2007

Aprovado em 04/Abr/2007 